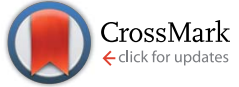

Cite this: RSC Adv., 2015, 5, 84865
Received 20th July 2015

Accepted 29th September 2015

DOI: $10.1039 / c 5 r a 14282 b$

\section{Optical performance improvement of semi- transparent metal film electrodes with biomimetic subwavelength gratings for high-performance optoelectronic device applications $\uparrow$}

\begin{abstract}
Minkyu Choi, Jung Woo Leem and Jae Su Yu*
We fabricated semi-transparent metal electrodes with biomimetic artificial antireflective subwavelength grating (SWG) structures at the surface of an ultraviolet (UV) curable polymer, i.e., Norland Optical Adhesive (NOA) 63, film on polyethylene terephthalate (PET) substrates for semi-transparent optoelectronic applications. The SWG patterns on the NOA63 film/PET were transferred from the silicon master molds with the conical SWG arrays by soft lithography. As a metal electrode, a silver (Ag) layer was deposited on the surface of the NOA63 SWG/PET substrates for different layer thicknesses. Compared with the Ag-coated flat bare PET (i.e., Ag/PET), the Ag/NOA63 SWG/PET showed a superior optical performance over a wide wavelength range of $400-1800 \mathrm{~nm}$. At the Ag layer thickness of $17 \mathrm{~nm}$, the Ag/NOA63 SWG/PET had lower average reflectance $\left(R_{\text {avg }}\right)$ and higher average transmittance $\left(T_{\text {avg }}\right)$ values of $\sim 25.1 \%$ and $\sim 33.9 \%$ than those (i.e., $R_{\text {avg }} \sim 55.2 \%$ and $T_{\text {avg }} \sim 26.4 \%$ ) of the Ag/PET, respectively, indicating the maximum figure of merit value of $10.23 \times 10^{-6} \mathrm{ohm}^{-1}$ (i.e., transmittance of 0.458 at a wavelength of $550 \mathrm{~nm}$ and sheet resistance of $39.71 \mathrm{ohm} \mathrm{sq}^{-1}$ ). Furthermore, the surface plasmon resonance phenomina (i.e., strong electric and magnetic fields) were observed around the surface of the Ag layer on NOA63 SWGs. Also, it exhibited less angle-dependent optical characteristics at light incident angles of $20-70^{\circ}$ under non-polarized light. For optical behaviors, theoretical analyses were also performed by a rigorous coupled-wave analysis method, which gives a similar tendency with the experimentally measured data.
\end{abstract}

\section{Introduction}

Transparent electrodes, which transmit light and transport electrical carriers simultaneously, have been widely utilized in various optoelectronic devices such as photovoltaics (PVs), light emitting diodes (LEDs), displays, touch panels, and transistors. ${ }^{1-4}$ Over the few past years, transparent conducting oxides (TCOs) including $\mathrm{SnO}_{2}: \mathrm{F}$ (FTO) and $\mathrm{In}_{2} \mathrm{O}_{3}: \mathrm{Sn}$ (ITO) have been commercially employed in devices due to their good optical and electrical properties (i.e., transmissions $>80 \%$ and resistivities < $10^{-3} \mathrm{ohm} \mathrm{cm}$ ). In particular, the ITO is the best TCO layer available today in high-performance applications. ${ }^{5,6}$ However, there has been an increasing demand in the development of transparent electrodes for an alternative of the ITO due to the increasing cost of indium (In) and the additional thermal process. Moreover, its high thickness disrupts a flexible device

Department of Electronics and Radio Engineering, Institute for Wearable Convergence Electronics, Kyung Hee University, 1732 Deogyeong-daero, Giheung-gu, Yongin-si 17104, South Korea. E-mail: jsyu@khu.ac.kr

$\dagger$ Electronic supplementary information (ESI) available: Absorption of the samples and calculated near-field intensity distribution of Ag/NOA63 SWG/PET at different wavelengths. See DOI: 10.1039/c5ra14282b approach. Many studies are reported on the various transparent electrodes such as conducting polymers, ${ }^{7}$ metal nanowire meshes, ${ }^{8}$ carbon-based materials (i.e., carbon nanotubes, graphenes, etc.), ${ }^{\mathbf{9}, \mathbf{1 0}}$ and oxide/metal/oxide hybrid electrodes (i.e., ITO/silver (Ag)/ITO), ${ }^{11}$ which have been mostly developed for semi-transparent organic device applications including organic PVs, LEDs, and transistors, which offer an extensive range of applications such as power-creation windows for buildings and automobiles, foldable devices, and other aesthetic architectural uses. $^{12,13}$ However, owing to the limitations including low throughput, device damages, and complexity in fabrication processes, it is necessary to develop the transparent electrodes in a more effective way including simple, low-damage, and mass-production processes. Recently, biomimetic antireflective nanostructures (e.g., subwavelength structures), which can efficiently reduce Fresnel reflection losses, have been actively studied using a soft lithographic pattern transfer technique. ${ }^{\mathbf{1 4 - 1 6}}$ The subwavelength structures form a linear effective gradientrefractive index profile that can effectively suppress the Fresnel reflection caused by the gap of indices between different media when the light propagates. ${ }^{17,18}$ In soft lithography, furthermore, once a mold is made, polymer gel is poured on it 
and cured via cross-linking. Removal of the mold results in a pattern successfully transferred onto a cross-linked polymer. ${ }^{19,20}$ This process can be repeated multiple times to fabricate multiple antireflective nanostructures, which would facilitate the mass-production of large-sized devices using rollto-roll and roll-to-plate processes. ${ }^{21}$ The most popular material used for soft lithography is polydimethylsiloxane (PDMS) due to its low free surface energy, flexibility, transparency, and hardness. ${ }^{22-24}$ For nanostructured replicas, ultraviolet (UV) curable polymers have been employed. Especially, a UV-curable Norland Optical Adhesive (NOA) 63 polymer is very suitable as an antireflective structured substrate because it has almost no absorption in the wide wavelength region of $400-1800 \mathrm{~nm}^{25}$ Besides, the nanopatterns are well formed using the soft lithography due to its high Young's modulus (1.655 GPa) including relatively good mechanical properties (i.e., hardness, robustness, and flexibility). ${ }^{26}$ In addition, it is also reported that there is no significant discoloration or deterioration of the NOA63 polymer films coated on glass or polyethylene terephthalate (PET) substrates, which were tested on $40 \mathrm{~W}$-mercury UV lamp exposure systems and heat treatments in an oven at temperatures of $<160{ }^{\circ} \mathrm{C} .{ }^{25}$ Therefore, it is very easy and simple to realize the cost-effective large-scalable transparent electrodes by coating a metal thin film onto antireflective nanostructured substrates prepared by the soft lithography. Moreover, the metal thin-film electrodes with periodic grating nanostructures, which strongly can trap the light, so-called surface plasmon resonance (SPR) effect, have been utilized for efficient light absorption in the active layer of optoelectronic devices (e.g., PVs and LEDs), resulting in the improvement of device performance. ${ }^{27-31}$ However, the optical performance of metal thin-film electrodes is strongly dependent on the film thickness. Thus, it is quite essential to investigate the optical behaviors of metalcoated antireflective nanostructured substrates for different metal film thicknesses including their electrical properties. In this work, we fabricated the semi-transparent electrodes by coating an $\mathrm{Ag}$ layer at the subwavelength grating surface of NOA63 polymer film on PET substrate (i.e., Ag/NOA63 SWG/ PET) via soft lithography and their optical and electrical properties were investigated, including SPR phenomena and incident light angle-dependent optical characteristics. For optical analyses of the samples, a rigorous coupled-wave analysis (RCWA) simulation was also performed.

\section{Experimental and numerical modelling details}

\section{Fabrication and characterization of Ag/NOA63 SWG/PET}

Fig. 1 shows the schematic diagram of the fabrication procedure for semi-transparent electrodes consisting of Ag/NOA63 SWG/PET. First, to fabricate the SWG patterned master molds, the single-polished (100) silicon (Si) was cleaned in acetone, methanol, and de-ionized water for $10 \mathrm{~min}$, sequentially. As an etch mask, the photoresist (PR) was spin-coated on the $\mathrm{Si}$ substrate. After the prebaking, the samples were exposed twice to the argon laser while rotating the sample by 60 degree,

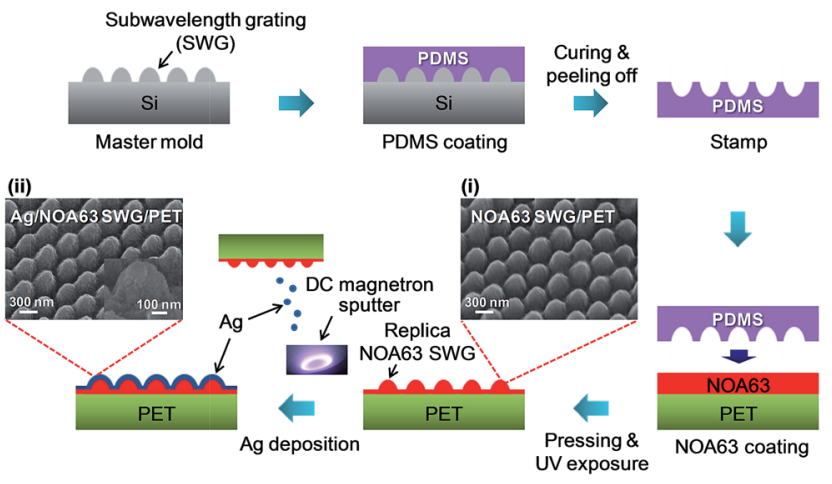

Fig. 1 Schematic diagram of the fabrication procedure for semitransparent electrodes consisting of Ag/NOA63 SWG/PET. $40^{\circ}$-tilted SEM images of (i) NOA63 SWG/PET and (ii) Ag/NOA63 SWG/PET.

followed by the development, creating two-dimensional (2D) hexagonal periodic dot PR arrays. Using the PR mask, finally, the SWG patterned Si molds were fabricated by inductively coupled plasma dry etching system, exhibiting the SWG height and period of $\sim 300 \mathrm{~nm}$ and $\sim 380 \mathrm{~nm}$, respectively. The details of the fabrication method were described in our previous work. $^{32}$ To transfer the SWG patterns onto the surface of PET substrates, the hard PDMS, formed by mixing and degassing four components (VDT-731, SIP 6831.1, SIT7900, and HMS-301 purchased from Gelest Inc.), was spin-coated on the Si mold and then cured in oven. Afterwards, the soft PDMS (base resin and curing agent, weight ratio $=10: 1$ ) was covered on the hard PDMS/Si mold and cured again. Lastly, the negative SWG patterned PDMS stamp was obtained by separating the PDMS film from the Si mold. The NOA63 polymer was spin-coated on PET substrates at $3000 \mathrm{rpm}$ for $1 \mathrm{~min}$, and then immediately the samples were pressed with $3.0 \mathrm{kgf}$ by the PDMS stamp. After UV exposure for $20 \mathrm{~min}$, the SWGs on the surface of NOA63/PET were formed by peeling off the PDMS stamp, creating the NOA63 SWG/PET. As shown in the scanning electron microscope (SEM) image of Fig. 1(i), it is observed that the SWGs with $2 \mathrm{D}$ hexagonal periodic pattern arrays were well transferred on the NOA63 film/PET substrates without critical deformations and cracks, having the SWG height and period of $\sim 300 \mathrm{~nm}$ and $\sim 380 \mathrm{~nm}$, respectively. After that, an Ag layer was deposited on the NOA63 SWG/PET by a DC magnetron sputtering system at 6 mTorr process pressure with $10 \mathrm{rpm}$ rotation speed at room temperature for different Ag layer thicknesses of 10, 13, 17, 20, 25 , and $35 \mathrm{~nm}$, respectively. The deposition rate and argon flow rate were kept to be $0.2 \mathrm{~nm} \mathrm{~s}^{-1}$ and $30 \mathrm{sccm}$, respectively. From the SEM image of Fig. 1(ii), the Ag layer was successfully deposited on the NOA63 SWG/PET. For comparison, the Ag layer was also prepared on the flat bare PET substrates for each $\mathrm{Ag}$ layer thickness. For structural and morphological properties of the samples, a SEM (LEO SUPRA 55, Carl Zeiss) system was used. Their optical behaviors were investigated by using a UVVis-NIR spectrophotometer (Cary 5000, Varian). A spectroscopic ellipsometry (V-VASE, J. A. Woollam) was used for the measurement of angle-dependent optical properties at incident angles of $20-70^{\circ}$ in non-polarized light. Electrical properties 
were also explored by using a Hall effect measurement system (Accent, HL5500PC).

\section{Numerical modelling and simulations}

Optical analyses (i.e., reflection, transmission, near-field intensity distribution) of the semi-transparent electrodes consisting of Ag/NOA63 SWG/PET structure were carried out using a RCWA method in a commercial software package (DiffractMOD, Rsoft Design Group). For the numerical modeling, the SWGs were simply represented by a periodic geometry with a scalar-valued function of three variables, $f(x, y, z)$, in a Cartesian coordinate system. Further details can be found in our previous work. ${ }^{30}$ The thicknesses of NOA63 polymer film and PET were set to be 1 and $200 \mu \mathrm{m}$, respectively. The refractive index and extinction coefficient of the PET and Ag used in these calculations were acquired from the index web site. ${ }^{33}$ For the NOA63 polymer film, the refractive index was assumed to be 1.56 while the extinction coefficient was not considered because it is negligible. ${ }^{25,34}$

\section{Results and discussion}

Fig. 2 shows the measured (i) reflectance and (ii) transmittance spectra of (a) Ag/PET and (b) Ag/NOA63 SWG/PET and the contour plots of variations of calculated (i) reflectance and (ii) transmittance spectra of (c) Ag/PET and (d) Ag/NOA63 SWG/PET for different $\mathrm{Ag}$ layer thicknesses in the wavelength $(\lambda)$ range of 400-1800 nm. As shown in Fig. 2, the optical behaviors of Ag thin-film electrodes strongly depend on the Ag layer thickness. As the Ag layer thickness is increased, the reflectance is increased while the transmittance is decreased. However, from Fig. 2(a) and (b), the Ag/NOA63 SWG/PET has the lower reflectance spectrum and the higher transmittance spectrum at each $\mathrm{Ag}$ layer thickness compared to the $\mathrm{Ag} / \mathrm{PET}$. In the optical simulation results of Fig. 2(c) and (d), similarly, the Ag/NOA63 SWG/PET also shows a superior optical performance over a wide wavelength range of $400-1800 \mathrm{~nm}$. This is attributed to the antireflective properties of the conical SWG structure on the surface NOA63 film/PET due to the linear effective gradient-refractiveindex profile via the SWG with a period of $380 \mathrm{~nm} .{ }^{32}$ Furthermore, the calculated results of both the samples with different Ag layer thicknesses exhibit a relatively reasonable agreement compared to the measured data in Fig. 2(a) and (b) though there is a slight discrepancy at some wavelengths. This may be the reason why it is difficult to exactly match the geometric simulation model to the experimentally fabricated structure and the mismatch of optical parameters (i.e., refractive index and extinction coefficient) for the constituent materials used in these experiments and calculations. The absorption spectra of both the samples were also estimated from 100 - reflectance - transmittance, as shown in Fig. S1 of the ESI. $\dagger$ Compared with the Ag/ PET, the Ag/NOA63 SWG/PET shows larger absorption. This is mainly the SPR effect (i.e., strong electric and magnetic fields) around the Ag layer on NOA 63 SWGs. ${ }^{27-31}$ The SPR effect of the Ag/NOA63 SWG/PET will be covered in Fig. 5.

Fig. 3 shows the estimated (a) average reflectance $\left(R_{\text {avg }}\right)$ and (b) average transmittance $\left(T_{\text {avg }}\right)$ values of $\mathrm{Ag} / \mathrm{PET}$ and $\mathrm{Ag} / \mathrm{NOA63}$
SWG/PET at $\lambda=400-1800 \mathrm{~nm}$. For the corresponding samples, the estimated $R_{\text {avg }}$ and $T_{\text {avg }}$ values at visible wavelengths of 400$700 \mathrm{~nm}$ are also shown in the insets of Fig. 3(a) and (b), respectively. As can be seen in Fig. 3, the estimated $R_{\text {avg }}$ and $T_{\text {avg }}$ values are increased and decreased with increasing the $\mathrm{Ag}$ layer thickness, respectively. For the Ag/PET, as the Ag layer thickness is increased from 10 to $35 \mathrm{~nm}$, the $R_{\text {avg }}$ and $T_{\text {avg }}$ values at $\lambda=$ $400-1800 \mathrm{~nm}$ are increased from 24.7 to $92.8 \%$ and reduced from 45.3 to $7.2 \%$, respectively. On the other hand, the Ag/NOA63 SWG/PET shows an improved optical property in both the reflectance and transmittance, exhibiting the variations from 12.2 to $53.5 \%$ in $R_{\text {avg }}$ and from 57.5 to $14.7 \%$ in $T_{\text {avg }}$, respectively. In the visible wavelength region of $400-700 \mathrm{~nm}$, the $\mathrm{Ag} /$ NOA63 SWG/PET has lower $R_{\text {avg }}$ values of $6.3-20.9 \%$ and higher $T_{\text {avg }}$ values of $48.4-31.5 \%$ compared to those (i.e., $R_{\text {avg }}=$ $26-80.6 \%$ and $T_{\text {avg }}=47.5-19.2 \%$ ) of the $\mathrm{Ag} / \mathrm{PET}$ at $\mathrm{Ag}$ layer thicknesses of 10-35 nm, respectively.

The electrical properties of Ag-coated nanostructured electrodes with different Ag layer thicknesses were also explored. Fig. 4 shows (a) sheet resistance $\left(R_{\mathrm{s}}\right)$, (b) transmittance at $\lambda=$ $550 \mathrm{~nm}$, and (c) figure of merit of Ag/PET and Ag/NOA63 SWG/ PET as a function of the Ag layer thickness. As expected, the $R_{\mathrm{S}}$ value of both the samples is enhanced with increasing the $\mathrm{Ag}$ layer thickness from 10 to $35 \mathrm{~nm}$. However, at the $\mathrm{Ag}$ layer thickness of $10 \mathrm{~nm}$, the Ag/PET shows a very poor electrical property while there are no measured data for the Ag/NOA63 SWG/PET due to the thin Ag layer thickness. On the contrary, at the relatively thick $\mathrm{Ag}$ layer thickness of $35 \mathrm{~nm}$, both the $\mathrm{Ag} /$ PET and Ag/NOA63 SWG/PET have good $R_{\mathrm{S}}$ values, exhibiting the $R_{\mathrm{S}}$ values of 1.45 and $6.77 \mathrm{ohm} \mathrm{sq.}{ }^{-1}$, respectively. For both the samples, the transmittance values at $\lambda=550 \mathrm{~nm}$ in Fig. 4(b) were obtained from the transmittance spectra in Fig. 2. The transmittance value is decreased with increasing the $\mathrm{Ag}$ layer thickness. However, for the Ag/NOA63 SWG/PET, its transmittance is less dependent on the Ag layer thickness compared to the Ag/PET, exhibiting that it shows the relatively small variation in the transmittance from $46.7 \%$ at $10 \mathrm{~nm}$ to $35 \%$ at 35 $\mathrm{nm}$ while the Ag/PET has a considerable change in the transmittance from $45.2 \%$ at $10 \mathrm{~nm}$ to $17.2 \%$ at $35 \mathrm{~nm}$. To determine the optimized condition among the Ag-coated nanostructured electrodes for different $\mathrm{Ag}$ layer thicknesses, the figure of merit ( $\left.\phi_{\mathrm{TC}}\right)$ was estimated using the $R_{\mathrm{S}}$ and transmittance. The $\phi_{\mathrm{TC}}$ is defined by Haacke as ${ }^{35}$

$$
\phi_{\mathrm{TC}}=\frac{T^{10}}{R_{\mathrm{s}}}
$$

where $T$ is the transmittance at $\lambda=550 \mathrm{~nm}$ and $R_{\mathrm{s}}$ is the sheet resistance of the samples. As shown in Fig. 4(c), both the samples show the maximum values at the $\mathrm{Ag}$ layer thickness of $17 \mathrm{~nm}$. However, for the Ag/NOA63 SWG/PET, the higher $\phi_{\mathrm{TC}}$ value of $10.23 \times 10^{-6} \mathrm{ohm}^{-1}$ was obtained by two parameters of $T=0.458$ and $R_{\mathrm{S}}=39.71 \mathrm{ohm} \mathrm{sq.}{ }^{-1}$ compared to that $\left(i . e ., \phi_{\mathrm{TC}}=\right.$ $9.08 \times 10^{-6} \mathrm{ohm}^{-1}, T=0.405$ and $\left.R_{\mathrm{s}}=13.08 \mathrm{ohm} \mathrm{sq.}^{-1}\right)$ of the Ag/PET. This Ag $(17 \mathrm{~nm}) /$ NOA63 SWG/PET sample has $T_{\text {avg }}$ values of 46.9 and $33.9 \%$ and $R_{\text {avg }}$ values of 9 and $25.1 \%$ at $\lambda=$ 400-700 $\mathrm{nm}$ and 400-1800 $\mathrm{nm}$, respectively, as can be seen in 

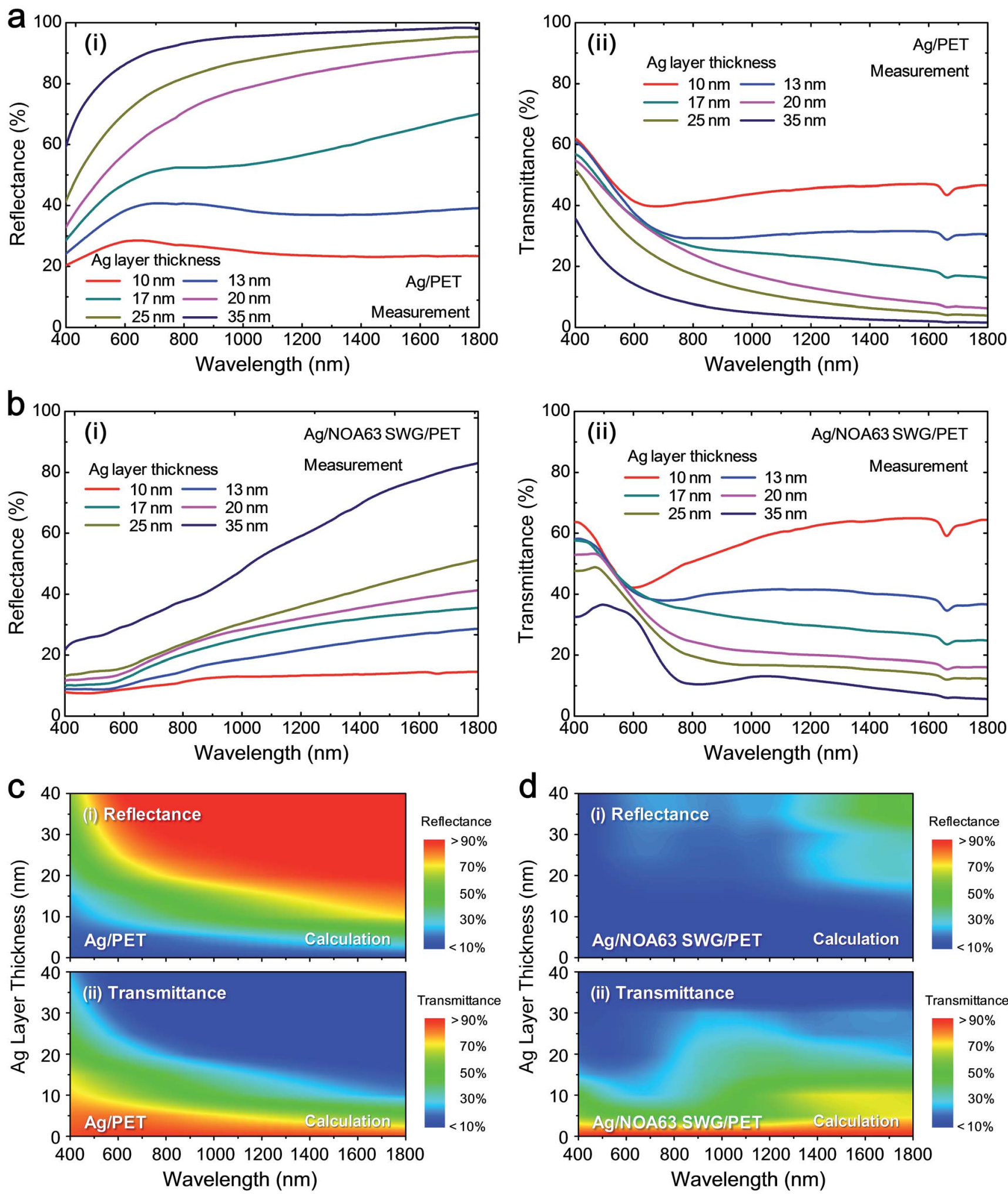

Fig. 2 Measured (i) reflectance and (ii) transmittance spectra of (a) Ag/PET and (b) Ag/NOA63 SWG/PET and contour plots of variations of calculated (i) reflectance and (ii) transmittance spectra of (c) Ag/PET and (d) Ag/NOA63 SWG/PET for different Ag layer thicknesses at $\lambda=400-$ $1800 \mathrm{~nm}$.

Fig. 3(b). Especially, at visible wavelengths of 400-700 $\mathrm{nm}$, this $T_{\text {avg }}$ (or $R_{\text {avg }}$ ) value is relatively low (or high) compared to the commercial ITO films on glasses (i.e., $T>80 \%, R_{\mathrm{S}}<15 \mathrm{ohm}$ sq. $\left.{ }^{-1}\right)^{36,37}$ and antireflective biomimetic nanostructured electrodes such as 3D ITO nanoparticles on ITO/AgO $/$ /ITO multilayer/PET (i.e., $\left.T=89 \%, R_{\mathrm{s}}=65 \mathrm{ohm} \mathrm{sq.}^{-1}\right)^{38}$ and ITO 

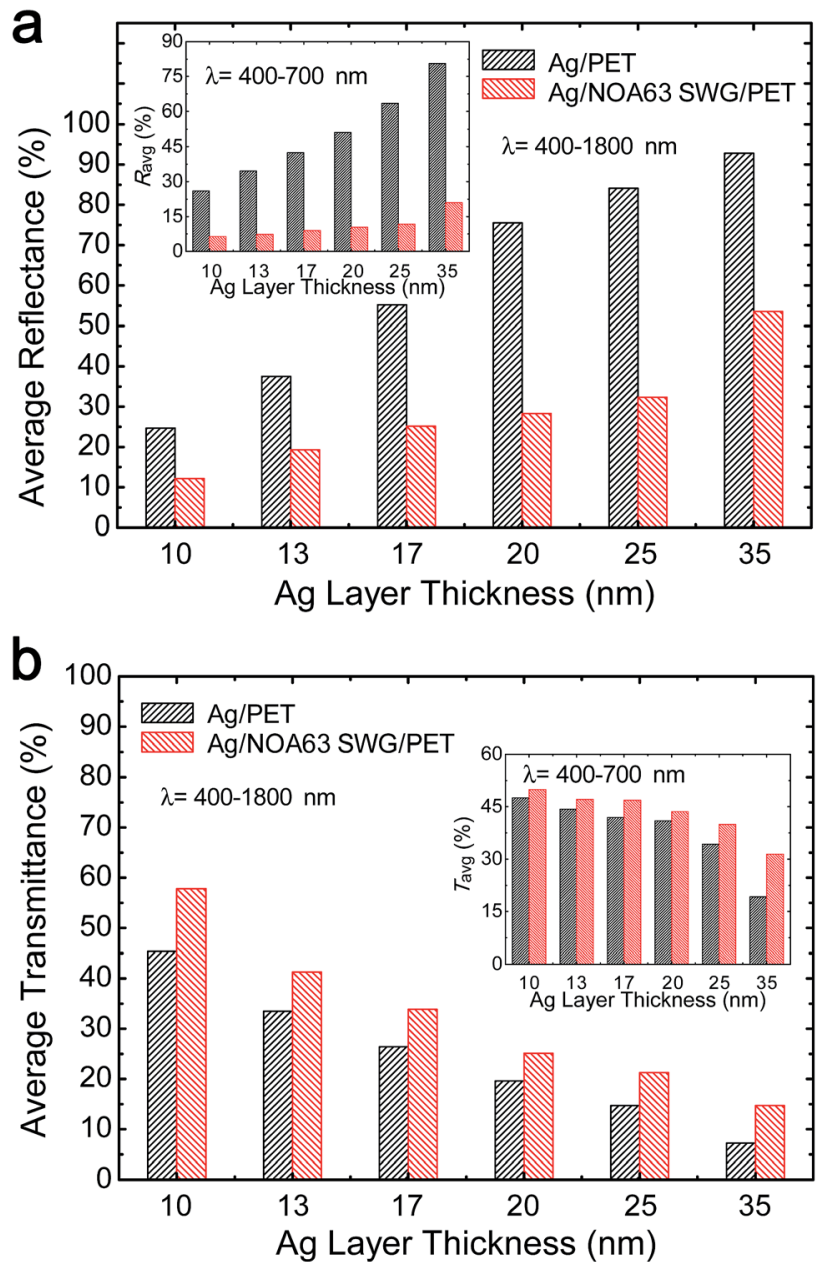

Fig. 3 Estimated (a) $R_{\text {avg }}$ and (b) $T_{\text {avg }}$ values of Ag/PET and Ag/NOA63 SWG/PET at $\lambda=400-1800 \mathrm{~nm}$. The $R_{\text {avg }}$ and $T_{\text {avg }}$ values of the corresponding samples at $\lambda=400-700 \mathrm{~nm}$ are also shown in the insets of (a) and (b), respectively.

nanocolumns/GaAs (i.e., $R<6 \%, R_{\mathrm{s}}=50 \mathrm{ohm} \mathrm{sq.} \cdot{ }^{-1}$ ). ${ }^{39}$ However, as mentioned above, the In is a rare earth metallic element, which causes the relatively high material cost of ITO. As an alternative of ITO, the antireflective biomimetic aluminumdoped zinc oxide (AZO) SWGs/film/Si structure was also reported in our group, exhibiting the $R_{\mathrm{avg}}=5 \%$ at $\lambda=300-1100$ $\mathrm{nm}$ and $R_{\mathrm{S}}$ values of $<30 \mathrm{ohm} \mathrm{sq.}{ }^{-1},{ }^{18}$ but the AZO SWGs/film electrodes were prepared by photolithography and dry etching processes with high fabrication cost and complexity. Furthermore, they also have large film thicknesses of $>400 \mathrm{~nm}$. These thick films are not suitable for flexible device applications since its brittleness causes cracks upon bending, including the additional thermal process at high temperatures of $>200-$ $300{ }^{\circ} \mathrm{C}$. On the other hand, herein, the Ag/NOA63 SWG/PET is easily prepared by facile soft lithography and sputtering methods which make mass production possible. Besides, its $R_{\mathrm{s}}$ value of $39.71 \mathrm{ohm} \mathrm{sq.}^{-1}$ is similar or lower compared to the other commercial ITO films and nanostructured electrodes. Also, its $T_{\text {avg }}$ value at $\lambda=400-700 \mathrm{~nm}$ is much higher than that
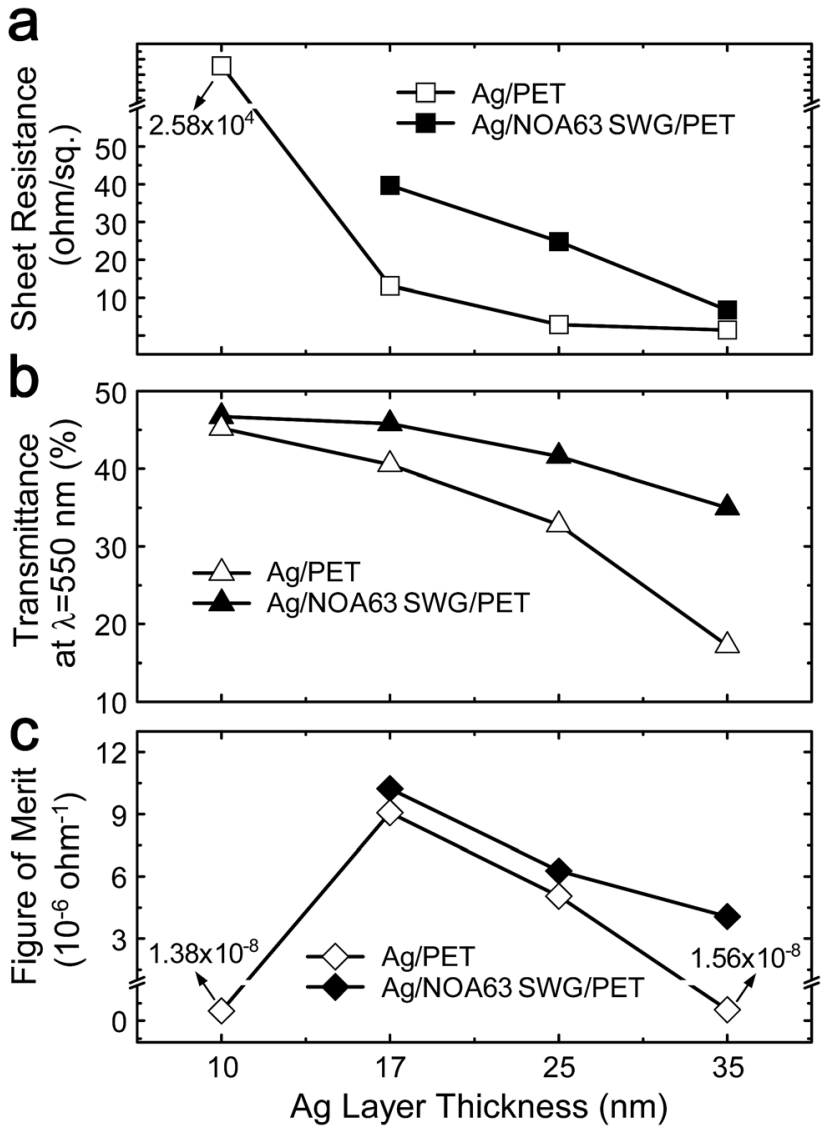

Fig. 4 (a) Sheet resistance, (b) transmittance at $\lambda=550 \mathrm{~nm}$, and (c) figure of merit of $\mathrm{Ag} / \mathrm{PET}$ and $\mathrm{Ag} / \mathrm{NOA63} \mathrm{SWG/PET}$ as a function of the Ag layer thickness.

(i.e., $T_{\mathrm{avg}}=25-30 \%$ ) of electrodes used in semi-transparent photovoltaic devices in previous literatures. ${ }^{\mathbf{4 0 - 4 2}}$

Fig. 5 shows (a) the photographs and (b) the contour plots of calculated electric $\left(E_{\mathrm{y}}\right)$ field intensity distributions of $\mathrm{Ag} / \mathrm{PET}$ and Ag/NOA63 SWG/PET at the Ag layer thickness of $17 \mathrm{~nm}$. As shown in Fig. 5(a), both the characters at the liquid crystal display (LCD) monitor and cellular phone camera are strongly reflected at the surface of the Ag/PET sample while there are almost no reflected ones on the surface of Ag/NOA63 SWG/PET sample. Additionally, the antireflective properties of SWGs can be also observed in the $E_{\mathrm{y}}$ field intensity distributions calculated by the RCWA simulation. In Fig. 5(b), for the Ag/PET, the $E_{\mathrm{y}}$ field intensity is high in the air region due to its strong reflection. For the Ag/NOA63 SWG/PET, on the other hand, there is a relatively weaker $E_{\mathrm{y}}$ field intensity in the air region (stronger $E_{\mathrm{y}}$ field intensity in the NOA63 region) due to its superior optical performance (i.e., antireflection) caused by the linear effective gradient-refractive-index profile via the SWG. Also, the metal thin-film electrodes with grating nanostructures can bring the enhanced light absorption in the active layer of optoelectronic devices (e.g., organic PVs or LEDs) due to the SPR effect (i.e., strong electric and magnetic fields) at the interface between the active and metal layers, which were generally proved by the calculated near-field intensity distributions in previous 
a
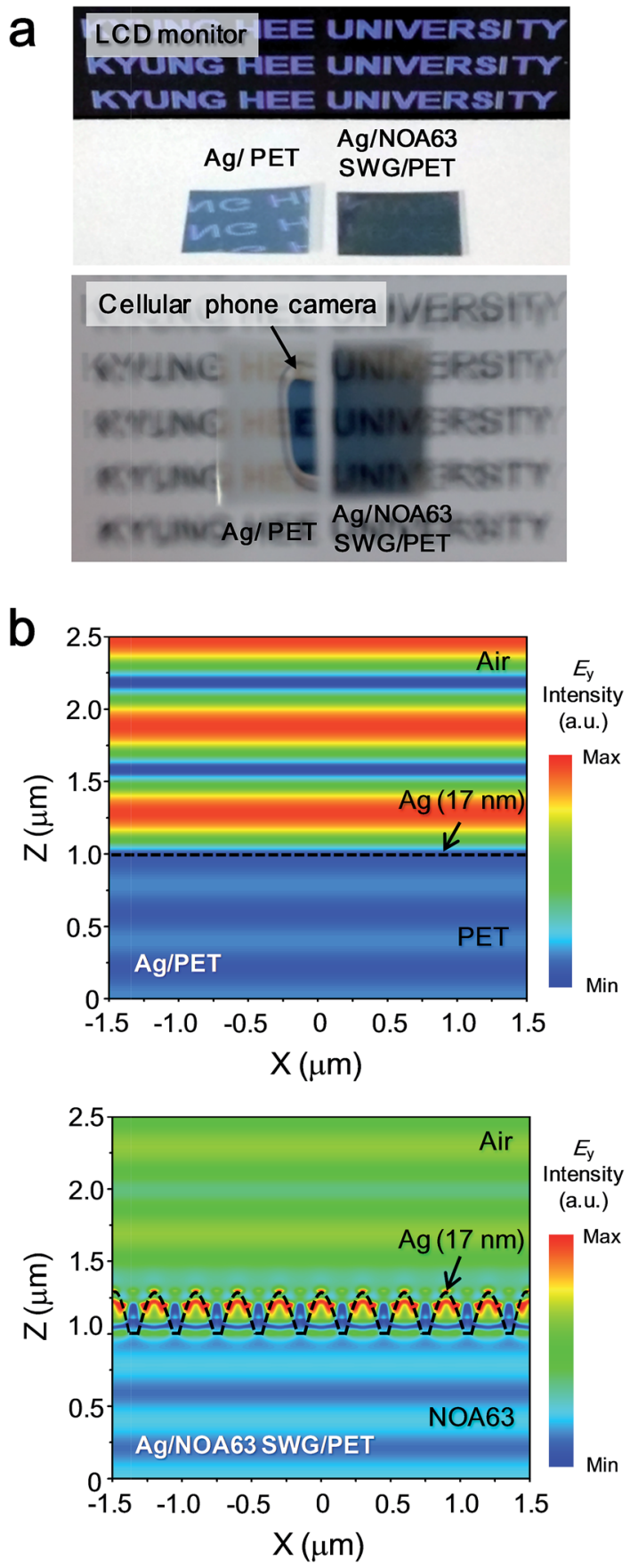

Fig. 5 (a) Photographs and (b) contour plots of calculated $E_{y}$ field intensity distributions of $\mathrm{Ag} / \mathrm{PET}$ and $\mathrm{Ag} / \mathrm{NOA63} \mathrm{SWG} / \mathrm{PET}$ at the $\mathrm{Ag}$ layer thickness of $17 \mathrm{~nm}$.

literatures. ${ }^{27-31}$ Similarly, the near-field intensity distributions of the Ag/NOA63 SWG/PET were calculated in Fig. S2 of the ESI. $\dagger$ The strong $E_{\mathrm{y}}$ or magnetic $\left(H_{\mathrm{y}}\right)$ fields around the $\mathrm{Ag}$ layer on NOA63 SWGs are observed at various wavelengths. Therefore, this SPR effect of Ag-coated NOA63 SWG electrodes can also boost the light absorption at the active layer in optoelectronic devices, thus leading to the enhancement of device performance.
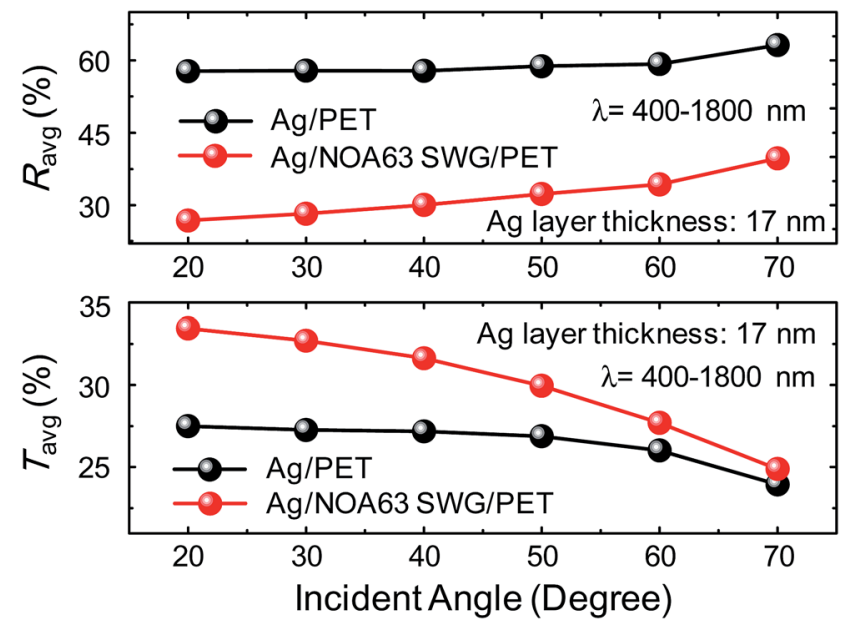

Fig. $6 R_{\text {avg }}$ and $T_{\text {avg }}$ values of Ag/PET and Ag/NOA63 SWG/PET at the Ag layer thickness of $17 \mathrm{~nm}$ as a function of light incident angle under non-polarized light.

The angle-dependent optical property is also an important parameter to achieve high-performance optical and optoelectronic devices. Fig. 6 shows the $R_{\text {avg }}$ and $T_{\text {avg }}$ values of Ag/PET and $\mathrm{Ag} / \mathrm{NOA63} \mathrm{SWG} / \mathrm{PET}$ at the $\mathrm{Ag}$ layer thickness of $17 \mathrm{~nm}$ as a function of light incident angle under non-polarized light. For both the samples, as the incident angle is increased, the $R_{\text {avg }}$ and $T_{\text {avg }}$ values are gradually increased and decreased, respectively. However, for the Ag/NOA63 SWG/PET, the lower $R_{\text {avg }}$ and higher $T_{\text {avg }}$ values are still maintained from 26.8 to $39.7 \%$ in $R_{\text {avg }}$ and from 33.4 to $24.9 \%$ in $T_{\text {avg }}$ compared to those (i.e., $R_{\mathrm{avg}}=$ $57.8-63.1 \%$ and $T_{\text {avg }}=27.5-23.9 \%$ ) of the $\mathrm{Ag} / \mathrm{PET}$ in an incident angle range from 20 to $70^{\circ}$, exhibiting the lower $R_{\mathrm{avg}}=31.9 \%$ and the larger $T_{\mathrm{avg}}=30 \%$ at $20-70^{\circ}$ (i.e., $R_{\mathrm{avg}}=59.1 \%$ and the $T_{\text {avg }}=26.5 \%$ for the $\left.\mathrm{Ag} / \mathrm{PET}\right)$, respectively.

\section{Conclusions}

The metal thin-film nanostructured electrodes consisting of the Ag/NOA63 SWG/PET were fabricated by the soft lithography via both the PDMS stamps and Si molds and the subsequent sputtering. The optical (i.e., reflection, transmission, and absorption) and electrical (i.e., sheet resistance) properties of the Ag/NOA63 SWG/PET were strongly dependent on the Ag layer thickness. The $\mathrm{Ag}(17 \mathrm{~nm}) / \mathrm{NOA63}$ SWG/PET with the maximum $\phi_{\mathrm{TC}}$ value of $10.23 \times 10^{-6} \mathrm{ohm}^{-1}$ (i.e., $T=0.458$ at $\lambda$ $=550 \mathrm{~nm}$ and $R_{\mathrm{s}}=39.71 \mathrm{ohm} \mathrm{sq.}^{-1}$ ) showed the lower $R_{\mathrm{avg}}$ and larger $T_{\text {avg }}$ values in the broad wavelength range of 400-1800 $\mathrm{nm}$, respectively. The theoretically calculated optical results showed a similar tendency to the experimentally measured data. The strong $E_{\mathrm{y}}$ or $H_{\mathrm{y}}$ fields (i.e., SPR effect) were observed around the Ag layer on NOA63 SWGs for different wavelengths. It also exhibited less-angle dependent optical characteristics compared to the Ag/PET at incident angles of $20-70^{\circ}$. These results can provide a better insight into the semi-transparent metal electrodes consisting of polymer SWGs, which can be simply prepared by the facile, mass-productive, and cost- 
effective soft lithography, with the broadband and wide-angle antireflection as well as the good electrical property including the SPR effect for high-performance optical and optoelectronic device applications.

\section{Acknowledgements}

This work was supported by the National Research Foundation of Korea (NRF) grant funded by the Korea government (MSIP) (No. 2014-069441).

\section{References}

1 C. C. Chen, L. Dou, J. Gao, W. H. Chang, G. Li and Y. Yang, Energy Environ. Sci., 2013, 6, 2714.

2 M. G. Kang and L. J. Guo, Adv. Mater., 2007, 19, 1391.

3 S. K. Chang-Jian, J. R. Ho and J. W. J. Cheng, Opt. Laser Technol., 2011, 43, 1371.

4 J. Lee, D. K. Hwang, J. M. Choi, K. Lee, J. H. Kim, S. Im, J. H. Park and E. Kim, Appl. Phys. Lett., 2005, 87, 023504.

5 W. Nie, H. Tsai, R. Asadpour, J. Blancon, A. J. Neukirch, G. Gupta, J. J. Crochet, M. Chhowalla, S. Tretiak, M. A. Alam, H. L. Wang and A. D. Mohite, Science, 2015, 347, 522.

6 Y. Yang, Y. Zheng, W. Cao, A. Titov, J. Hyvonen, J. R. Manders, J. Xue, P. H. Holloway and L. Qian, Nat. Photonics, 2015, 9, 259.

7 B. Winther-Jensen and F. C. Krebs, Sol. Energy Mater. Sol. Cells, 2006, 90, 123.

8 L. Hu, H. S. Kim, J. Y. Lee, P. Peumans and Y. Cui, ACS Nano, 2010, 4, 2955.

9 Y. H. Kim, L. Müller-Meskamp, A. A. Zakhidov, C. Sachse, J. Meiss, J. Bikova, A. Cook, A. A. Zakhidov and K. Leo, Sol. Energy Mater. Sol. Cells, 2012, 96, 244.

10 Y. Y. Lee, K. H. Tu, C. C. Yu, S. S. Li, J. Y. Hwang, C. C. Lin, K. H. Chen, L. C. Chen, H. L. Chen and C. W. Chen, ACS Nano, 2011, 5, 6564.

11 M. Fahland, P. Karlsson and C. Charton, Thin Solid Films, 2001, 392, 334.

12 K. Ellmer, Nat. Photonics, 2012, 6, 809.

13 D. S. Hecht, L. Hu and G. Irvin, Adv. Mater., 2011, 23, 1482. 14 F. Galeotti, F. Trespidi, G. Timò and M. Pasini, ACS Appl. Mater. Interfaces, 2014, 6, 5827.

15 K. Forberich, G. Dennler, M. C. Scharber, K. Hingerl, T. Fromherz and C. J. Brabec, Thin Solid Films, 2008, 516, 7167.

16 D. H. Ko, J. R. Tumbleston, K. J. Henderson, L. E. Euliss, J. M. DeSimone, R. Lopez and E. T. Samulski, Soft Matter, 2011, 7, 6404.

17 D. G. Stavenga, S. Foletti, G. Palasantzas and K. Arikawa, Proc. R. Soc. B, 2006, 273, 661.

18 J. W. Leem, D. H. Joo and J. S. Yu, Sol. Energy Mater. Sol. Cells, 2011, 95, 2221.
19 Y. Xia and G. M. Whitesides, Annu. Rev. Mater. Sci., 1998, 28, 153.

20 T. W. Odom, J. C. Love, D. B. Wolfe, K. E. Paul and G. M. Whitesides, Langmuir, 2002, 18, 5314.

21 S. H. Ahn and L. J. Guo, ACS Nano, 2009, 3, 2304.

22 Y. M. Song, Y. Xie, V. Malyarchuk, J. Xiao, I. Jung, K. J. Choi, Z. Liu, H. Park, C. Lu, R. H. Kim, R. Li, K. B. Crozier, Y. Huang and J. A. Rogers, Nature, 2013, 497, 95.

23 J. W. Leem, M. Choi and J. S. Yu, ACS Appl. Mater. Interfaces, 2015, 7, 2349.

24 C. S. Huang, E. Y. B. Pun and W. C. Wang, J. Opt. Soc. Am. B, 2009, 26, 1256.

25 J. W. Leem, X. Y. Guan, M. Choi and J. S. Yu, Sol. Energy Mater. Sol. Cells, 2015, 134, 45.

26 S. Y. Heo, J. K. Koh, G. Kang, S. H. Ahn, W. S. Chi, K. Kim and J. H. Kim, Adv. Energy Mater., 2014, 4, 1300632.

27 X. H. Li, W. E. I. Sha, W. C. H. Choy, D. S. S. Fung and F. X. Xie, J. Phys. Chem. C, 2012, 116, 7200.

28 X. Li, W. C. H. Choy, L. Huo, F. Xie, W. E. I. Sha, B. Ding, X. Guo, Y. Li, J. Hou, J. You and Y. Yang, Adv. Mater., 2012, 24, 3046.

29 J. D. Chen, L. Zhou, Q. D. Ou, Y. Q. Li, S. Shen, S. T. Lee and J. X. Tang, Adv. Energy Mater., 2014, 4, 1301777.

30 J. W. Leem, S. Kim, C. Park, E. Kim and J. S. Yu, ACS Appl. Mater. Interfaces, 2015, 7, 6706.

31 Y. G. Bi, J. Feng, Y. F. Li, X. L. Zhang, Y. F. Liu, Y. Jin and H. B. Sun, Adv. Mater., 2013, 25, 6969.

32 J. W. Leem, Y. M. Song, Y. T. Lee and J. S. Yu, Appl. Phys. B, 2010, 100, 891.

33 Filmetrics Refractive index database (2015), retrieved 2015, http://www.filmetrics.com.

34 Norland Optical Adhesive (2015), retrieved 2015, https:// www.norlandprod.com.

35 G. Haacke, J. Appl. Phys., 1976, 47, 4086.

36 D. Paeng, J. H. Yoo, J. Yeo, D. Lee, E. Kim, S. H. Ko and C. P. Grigoropoulos, Adv. Mater., 2015, 27, 2762.

37 R. Pandey, C. H. Wie, X. Lin, J. W. Lim, K. K. Kim, D. K. Hwang and W. K. Choi, Sol. Energy Mater. Sol. Cells, 2015, 134, 5 .

38 W. Wang, T. S. Bae, Y. H. Park, D. H. Kim, S. Lee, G. Min, G. H. Lee, M. Song and J. Yun, Nanoscale, 2014, 6, 6911.

39 P. Yu, C. H. Chang, C. H. Chiu, C. S. Yang, J. C. Yu, H. C. Kuo, S. H. Hsu and Y. C. Chang, Adv. Mater., 2009, 21, 1618.

40 C. Roldán-Carmona, O. Malinkiewicz, R. Betancur, G. Longo, C. Momblona, O. Malinkiewicz, R. Betancur, G. Longo and C. Momblona, Energy Environ. Sci., 2014, 7, 2968.

41 K. S. Chen, J. F. Salinas, H. L. Yip, L. J. Huo, J. H. Hou and A. K. Y. Jen, Energy Environ. Sci., 2012, 5, 9551.

42 C. C. Chueh, S. C. Chien, H. L. Yip, J. F. Salinas, C. Z. Li, K. S. Chen, F. C. Chen, W. C. Chen and A. K. Y. Jen, Adv. Energy Mater., 2013, 3, 417. 